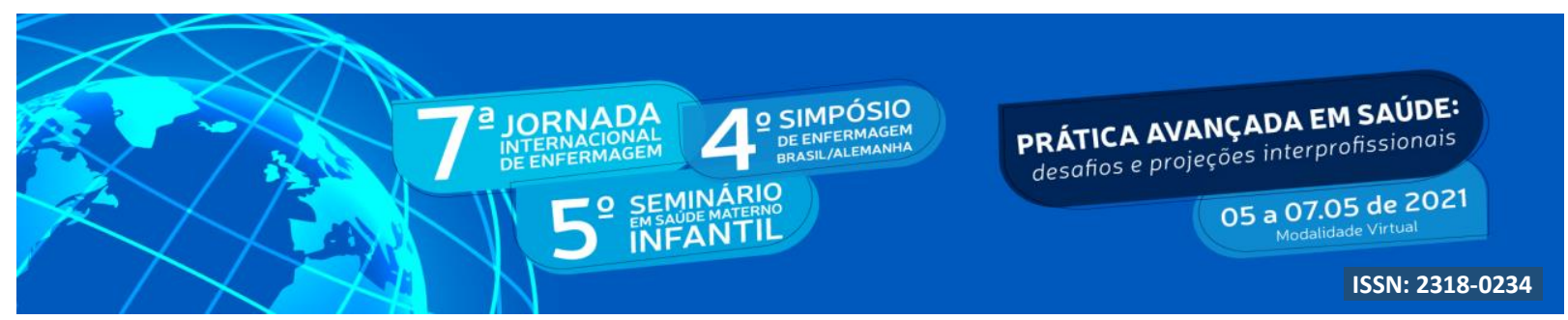

\title{
INTERNAÇÕES EM UNIDADE CANGURU DE HOSPITAL DE REFERENCIA PARA ALTO RISCO MATERNO E INFANTIL
}

\section{Michelle Thais Migoto ${ }^{1}$ Márcia Helena de Souza Freire ${ }^{2}$; Francielle de Souza Ulbrich ${ }^{3}$; Alessandra Patrícia Stelmak ${ }^{4}$; Gabrielle Freitas Saganski ${ }^{5}$.}

\section{RESUMO}

Objetivo: caracterizar as internações ocorridas em Unidade Canguru em um hospital de ensino, referência para o alto risco materno e infantil, do estado do Paraná. Método: estudo transversal, descritivo, de abordagem quantitativa, desenvolvido com dados secundários, do Sistema de Informação Hospitalar e Resumo de Alta, em 2016. Resultados: Das 177 internações identificadas, observou-se prevalência do sexo masculino (50,9\%); maior proporção de prematuros $(74,0 \%)$, com baixo peso ao nascer $(59,4 \%)$. Ocorreu alta médica hospitalar para domicílio para $84,8 \%$ da população. Quanto a procedência materna, $94,8 \%$ da $2^{\text {a }}$ Regional de Saúde, a Metropolitana, houve perdas de variáveis maternas, devido não preenchimento, a saber: a escolaridade, estado civil e número de consultas de pré-natal, para $51,0 \%, 41,2 \%$ e $29,4 \%$ dos dados, respectivamente. Conclusão: $O$ perfil dos neonatos internados em Unidade Canguru, apontou para adequabilidade à aplicação do Método Canguru. Recomenda-se melhor gestão das informações registradas, para maior segurança do paciente, profisssional de saúde e instituição.

Descritores: Enfermagem Neonatal; Método Canguru; Unidade de Terapia Intensiva Neonatal; Recém-nascido; Enfermagem materno-infantil.

\begin{abstract}
Objecive: to characterize the hospitalizations that occurred in the Kangaroo Unit in 2016, in a referral hospital for high maternal and infant risk. Methods: pesquisa transversal, descritiva, de abordagem quantitativa, desenvolvida a partir de dados secundários, do Sistema de Informação Hospitalar e resumo de alta. Results: Of the 177 hospitalizations, male prevalence was observed (50.9\%); a higher proportion of preterm infants (74.0\%), with low birth weight $(59.4 \%) .84 .8 \%$ of the population was hospitalized. Regarding maternal origin, 94.8\% came from the second Regional Health; there were losses of maternal variables, such as schooling, marital status, and several prenatal consultations, in $51.0 \%, 41.2 \%$ and $29.4 \%$ of the data, respectively. Conclusion: The profile of mothers and newborns admitted to the Kangaroo Unit points to appropriate clientele for the application of the Kangaroo Method. Recommended better management of the information recorded, for greater safety to the patient, the health professional, and the institution.
\end{abstract}

Keywords: Neonatal Nursing; Kangaroo-Mother Care Method; Intensive Care Units, Neonatal; Infant, Newborn; Maternal-Child Nursing.

\section{INTRODUÇÃO}

O nascimento prematuro é um problema global de saúde pública, corresponsável pela

\footnotetext{
${ }^{1}$ Enfermeira. Doutoranda no Programa de Pós-Graduação em Enfermagem. UFPR. E-mail: michellemigoto@gmail.com

${ }^{2}$ Orientadora. Doutora em Saúde Pública. Departamento de Enfermagem UFPR. E-mail: marciahelenafreire@gmail.com

${ }^{3}$ Enfermeira. Universidade Federal do Paraná (UFPR). E-mail: fsouza952@gmail.com

${ }^{4}$ Enfermeira. Mestre pelo Programa de Pós-Graduação em Enfermagem Profissional. UFPR. E-mail: apstelmak@gmail.com

${ }^{5}$ Enfermeira. Doutoranda no Programa de Pós-Graduação em Enfermagem. UFPR. E-mail: gabisaga@gmail.com
} 


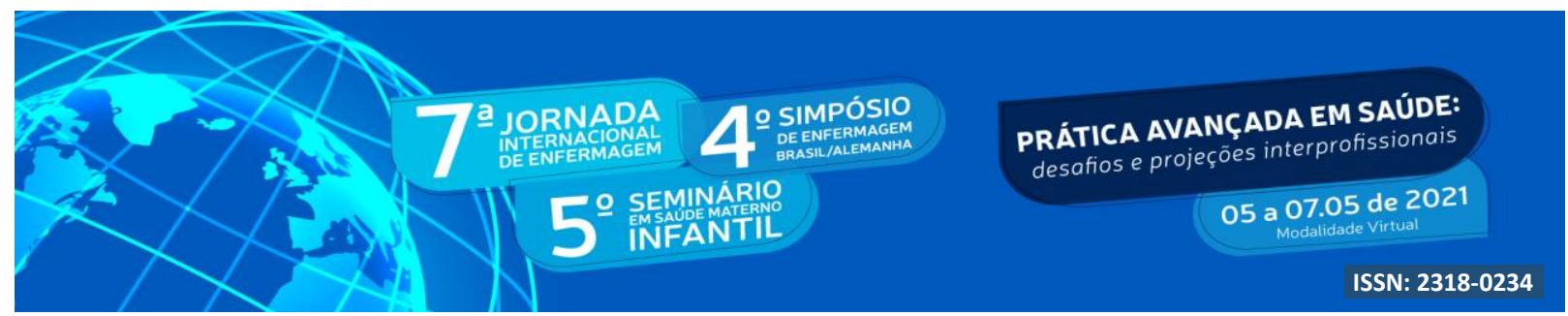

crescente ocorrência dos óbitos na primeira semana de vida (WHO, 2012), por isso aprimorar a qualidade da assistência é essencial para a manutenção da vida desta população (STELMAK; et al., 2017). Consideram-se prematuros os recém-nascidos com idade gestacional inferior a 37 semanas, subdivididos em três classificações: prematuros extremos, os menores de 28 semanas; muito prematuros, entre 28 a 32 semanas; e, os prematuros moderados ou tardios, nascidos após 32 semanas (WHO, 2012).

A assistência à saúde desta população precisa considerar a humanização, para o fortalecimento do vínculo entre o recém-nascido e sua família. Nesta perspectiva, foi estabelecido o Método Canguru que reúne estratégias de cuidado com intervenções que favorecem o seu desenvolvimento com qualidade de vida (BRASIL, 2011). Realizado em três fases: 1) Na Unidade de Terapia Intensiva Neonatal (UTIN), fortalecendo o vínculo, com ampliação do acesso aos pais e, apoiado em intensa orientação no contexto de internação (BRASIL, 2011); 2) Na Unidade de Cuidados Intermediários (UINCa) a mãe retorna ao ambiente hospitalar, em tempo integral, e cuida do recém-nascido com o apoio da equipe multiprofissional (BRASIL, 2011); 3) No acompanhamento ambulatorial após a alta em tempo oportuno, avaliando o crescimento e a adaptação domiciliar para evitar a reinternação e encaminhar as especialidades quando necessário (BRASIL, 2011).

Dependendo da classificação da prematuridade e do peso ao nascer, o tempo de internação destes recém-nascidos em UTIN poderá variar entre 2 à 50 dias (SOUSA; et al., 2017). Com base no exposto, os benefícios deste modelo de cuidado promoveram sua expansão em todo o território nacional. Sendo imprescindível para a gestão e aperfeiçoamento deste modelo de cuidado humanizado a contínua produção de evidências científicas.

\section{OBJETIVO}

Caracterizar as internações ocorridas em Unidade de Cuidados Intermediários Canguru, de um hospital de ensino, referência para o alto risco materno e infantil, no Paraná.

\section{METODOLOGIA}

Trata-se de um estudo quantitativo, transversal e descritivo (ARAGÃO, 2011), realizado em uma UCINCa, de um hospital escola, em Curitiba, Paraná, em 2016. Ele conta com 10 leitos intensivos, 15 semi-intensivos e 5 de UCINCa. É referência para os municípios 


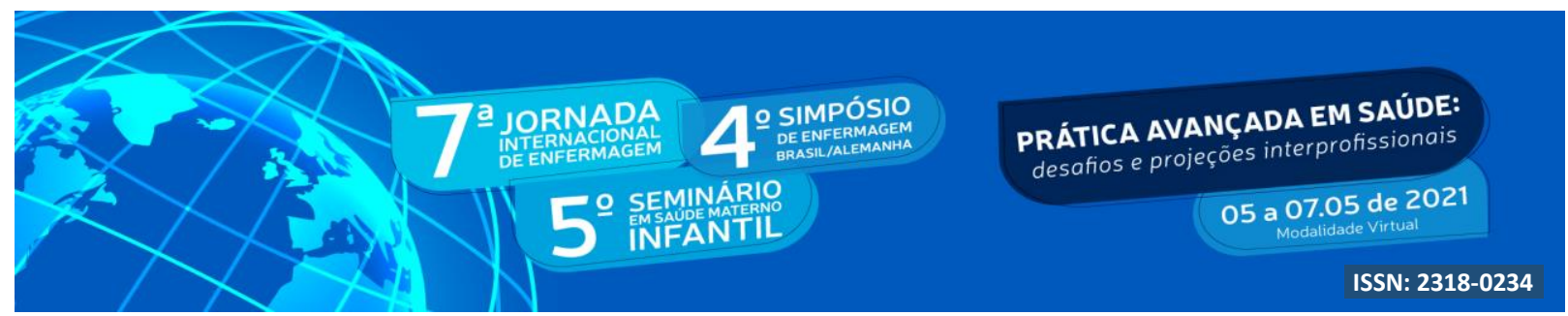

do estado do Paraná, vinculada ao Sistema Único de Saúde, além de manter especialidades ambulatoriais, clínicas e cirúrgicas referenciadas para todas as regiões do Brasil.

Foi utilizado dados secundários do Sistema de Informação Hospitalar (SIH), no qual armazena dados sobre os internamentos, e também, dados do resumo de alta que foi preenchido pela equipe médica, que contém dados não disponibilizados no SIH. A população foi composta por recém-nascidos internados UCINCa, entre 01 de janeiro a 31 de dezembro de 2016.

Trabalharam-se com as variáveis do recém-nascido: sexo, idade gestacional, peso ao nascer, peso na alta, motivo da internação, destino e período de internação. As variáveis maternas: idade, escolaridade, estado civil e tipo de parto. Para organização dos dados utilizou-se o Microsoft Office Excel®, aplicando a estatística descritiva com frequência, proporção e mediana para a análise dos dados.

Foi utilizado o checklist Strengthening the Reporting of Observational studies in Epidemiology (STROBE) para a elaboração desta pesquisa (MALTA; et al., 2010). Respeitou-se a Resolução ${ }^{\circ} 466$ de 2012 (BRASIL, 2012), a pesquisa foi aprovada pelo Comitê de Ética em Pesquisa da Universidade Federal do Paraná, sob o parecer n ${ }^{\circ}$ 1.061.330.

\section{RESULTADOS E DISCUSSÃO}

Foram identificados 1.577 nascimentos, em 2016, destes $27,3 \%$ foram admitidos no serviço de Unidade Neonatal. Totalizaram-se 177 internações na UCINCa, destes, 56,5\% eram filhos de mulheres com idade entre 20 a 34 anos, que passaram por uma mediana de 9 consultas de pré-natal; $87,0 \%$ foram produtos de gestações únicas e, 61,6\% nasceram de parto cirúrgico (Tabela 1). As variáveis maternas relacionadas à escolaridade, estado civil e número de consultas de pré-natal apresentaram grande proporção de perda de informação, devido o não preenchimento das mesmas, $51,0 \%, 41,2 \%$ e 29,4\%, respectivamente (Tabela 1).

Quanto as variáveis dos recém-nascidos observaram-se discreta prevalência do sexo masculino (50,9\%), e maior proporção de prematuros $(74,0 \%)$ com idade gestacional entre 32 a $<37$ semanas $(45,8 \%)$. Em relação ao peso, 59,4\% apresentaram baixo peso ao nascer, destacaram-se os nascidos com peso entre 1.500 a $2.499 \mathrm{~g}$ (36,7\%), entretanto classificados com peso adequado a idade gestacional $(63,8 \%)$, com o percentil entre 10 a 90 . Destaca-se que a mediana do peso, no valor de $2.050 \mathrm{~g}$, está na faixa do baixo peso ao nascer, de modo 


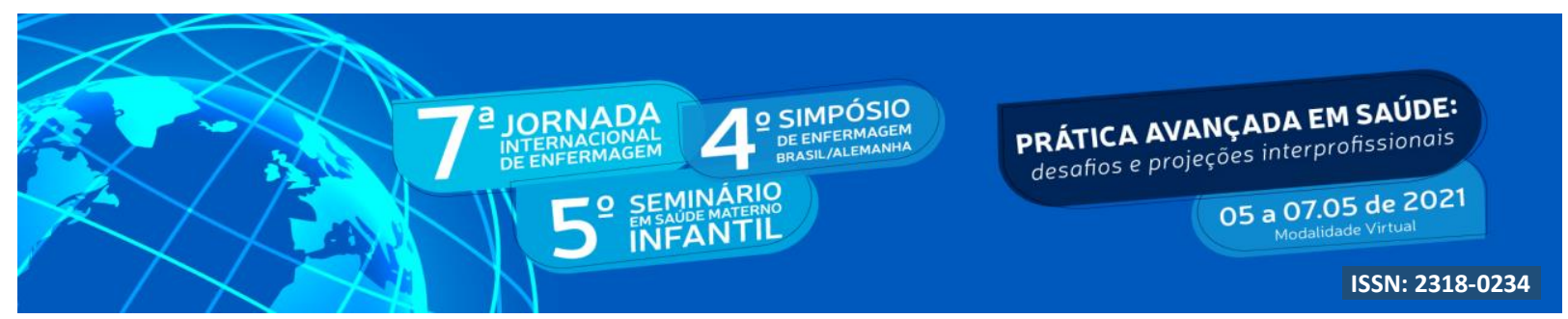

que $50 \%$ dos neonatos internados na UCINCa se enquadram na categoria do baixo peso ao nascer (Tabela 1). Em relação à idade cronológica no momento da internação na Unidade Canguru, sobressaíram-se os recém-nascidos entre 7 a 28 dias de vida (59,3\%), com tempo médio de internação de 6 dias, e mediana de 4 dias, com variação entre 1 a 39 dias. Assim, $50 \%$ dos neonatos permanecem até 4 dias em Unidade Canguru. Apresentaram como desfecho a alta médica, $84,8 \%$ das crianças (Tabela 1 ).

Tabela 1: Variáveis dos Recém-Nascidos Internados em Unidade de Cuidados Intermediários Canguru segundo as Características Maternas e do Recém-Nascido, Hospital de Ensino, Curitiba, PR, Brasil, 2016.

\begin{tabular}{|c|c|c|c|}
\hline Variáveis & $\mathbf{N}$ & $\%$ & MEDIANA \\
\hline \multicolumn{4}{|c|}{ Variáveis Maternas } \\
\hline Idade da mãe (anos) & & & - \\
\hline Até 19 & 28 & 15,9 & \\
\hline De 20 a 34 & 100 & 56,5 & \\
\hline Igual ou maior que 35 & 39 & 22,0 & \\
\hline Não informado & 10 & 5,6 & \\
\hline \multicolumn{4}{|l|}{ Escolaridade } \\
\hline Fundamental Completo & 10 & 5,6 & \\
\hline Fundamental Incompleto & 10 & 5,6 & \\
\hline Médio Completo & 41 & 23,2 & \\
\hline Médio Incompleto & 13 & 7,3 & \\
\hline Superior Completo & 9 & 5,1 & \\
\hline Superior Incompleto & 3 & 1,7 & \\
\hline Técnico & 1 & 0,5 & \\
\hline Não informado & 90 & 51,0 & \\
\hline \multicolumn{3}{|l|}{ Estado Civil } & - \\
\hline Casada & 53 & 30,0 & \\
\hline Separada & 2 & 1,1 & \\
\hline Solteira & 19 & 10,7 & \\
\hline União Estável & 30 & 17,0 & \\
\hline Não informado & 73 & 41,2 & \\
\hline \multicolumn{3}{|l|}{ Consultas de Pré-Natal } & 9 \\
\hline$<7$ & 31 & 17,5 & \\
\hline$\geq 7$ & 94 & 53,1 & \\
\hline Não informado & 52 & 29,4 & \\
\hline \multicolumn{3}{|l|}{ Tipo de Gestação } & - \\
\hline Única & 154 & 87,0 & \\
\hline Gemelar & 23 & 13,0 & \\
\hline \multicolumn{3}{|l|}{ Tipo de Parto } & - \\
\hline Via cirúrgica & 109 & 61,6 & \\
\hline Normal & 61 & 34,5 & \\
\hline \multirow[t]{2}{*}{ Não informado } & 7 & 3,96 & \\
\hline & \multicolumn{2}{|c|}{ Variáveis do recém-nascido } & \\
\hline Sexo & & & - \\
\hline Masculino & 90 & 50,9 & \\
\hline Feminino & 87 & 49,1 & \\
\hline Idade Gestacional & & & - \\
\hline
\end{tabular}




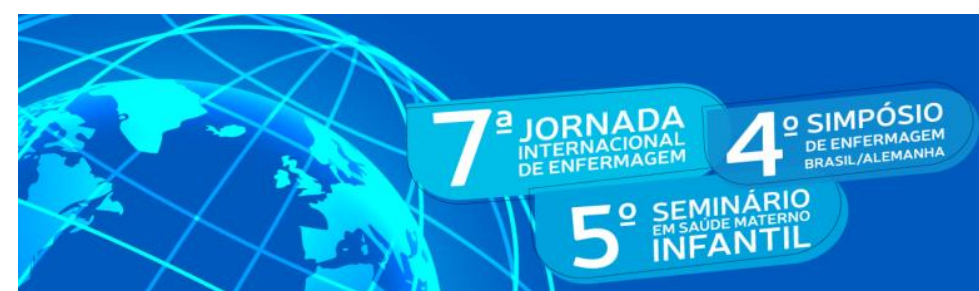

PRÁTICA AVANÇADA EM SAÚdE:

projeçőes interprofissionais

05 a 07.05 de 2021

$\leq 28$ (Prematuro Extremo)

$28<32$ (Muito Prematuro)

$32<37$ (Prematuro Moderado ou tardio)

$37<42$ (Termo)

Não informado

Classificação do $\mathbf{R N}$

RNPT

RNT

Não informado

Classificação em Peso

Peso de nascimento

$<1000$

$1000-1499$

$1500-2499$

2500-3999

$\geq 4000$

Não informado

Classificação em Percentil

PIG

AIG

GIG

Não informado

Idade de internação na UCINCa

Neonatal precoce (0-6 dias)

Neonatal tardio (7-28 dias)

Pós-neonatal (29-364 dias)

\section{Peso de Alta}

1800-2500

2501-3999

$\geq 4000$

Não informado

Destino

Transferência

Alta Médica
19

27

81

40

10

131

42

4

17

65

59

7

6

25

113

22

17

28

105

44

87

52

29

27

150
10,7

15,2

45,8

22,7

5,6

74,0

23,7

2,3

13,0

9,7

36,7

33,3

3,9

3,4

14,1

63,8

12,4

9,7

15,8

59,3

24,9

49, 1

29,4

5,1

16,4

15,2

84,8

Fonte: dados do SIH (2016).

2050

Com relação ao peso de alta hospitalar identificou-se que 34,5\% saíram com peso acima de $2.500 \mathrm{~g}$, entretanto, para 16,4\% não foram informados os pesos de alta no Resumo de Alta, documento contido no prontuário, e ainda, quase $50 \%$ dos neonatos, foram para seus domicílios com baixo peso ao nascer (menor do que 2.500g). Durante o ano de 2016, ocorreram apenas internações de procedentes do estado do Paraná. Em relação à procedência materna, foi observada maior prevalência do município de Curitiba $(64,4 \%)$ e, a seguir, Colombo (6,7\%) e Pinhais (5,0\%). Quando classificada em Regionais de Saúde, evidencia-se predominância da $2^{\text {a }}$ Regional de Saúde, a Metropolitana, (94,8\%), seguido pela $1^{\text {a }}$ Regional de Saúde, a de Paranaguá $(2,9 \%)$.

A Tabela 2 detalha informações referentes ao diagnóstico principal confirmado de 


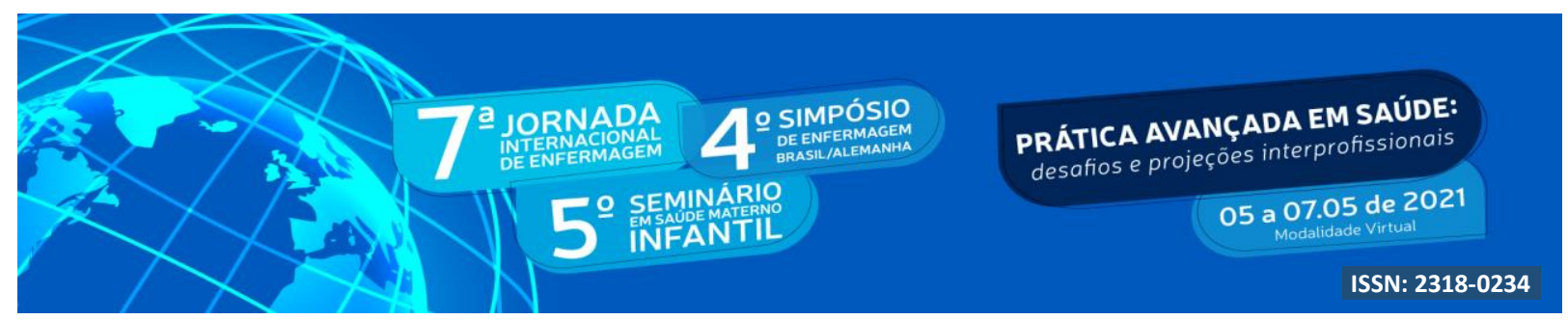

internação, assim denominado pelo SIH, totalizaram-se 190 diagnósticos principais confirmados, detalhado nos diagnósticos prevalentes, com maior número de registros. Assim, ressalta-se que a prevalência $(62,1 \%)$ foi das internações relacionadas ao Capítulo XVI Algumas afecções originadas no período perinatal e, deste sobretudo, os Transtornos relacionados com a gestação de curta duração e peso baixo ao nascer não classificados em outra parte $(44,0 \%)$.

Tabela 4: Diagnóstico Principal das Internações de Recém-nascidos em Unidade de Cuidados Intermediários Canguru, Curitiba, PR, Brasil, 2016.

\begin{tabular}{l|r|c}
\hline \multicolumn{1}{c|}{ CLASSIFICAÇÃO INTERNACIONAL DE DOENÇAS } & N & \% \\
\hline Capítulo XVI - Algumas afecções geradas no período perinatal (P00-P96) & $\mathbf{1 1 8}$ & $\mathbf{6 2 , 1}$ \\
P00 Feto e recém-nascidos afetados por afecções maternas, não obrigatoriamente & 3 & 1,57 \\
relacionadas com a gravidez atual & & \\
P05 Feto e recém-nascido afetados por traumatismo materno & 1 & 0,52 \\
P07 Transtornos relacionados com a gestação de curta duração e peso baixo ao nascer & 84 & 44,0 \\
não classificados em outra parte & 5 & 2,63 \\
P08 Feto e recém-nascido afetados por outras afecções maternas & 11 & 5,78 \\
P22 Desconforto respiratório do recém-nascido & 2 & 1,05 \\
P28 Outras afecções respiratórias originadas no período perinatal & 3 & 1,57 \\
P37 Outras doenças infecciosas e parasitárias congênitas & 3 & 1,57 \\
P59 Icterícia neonatal devida a outras causas e as não especificadas & 6 & 3,41 \\
Outro & $\mathbf{1 1}$ & $\mathbf{5 , 7 9}$ \\
\hline Capitulo XVIII - Sintomas, sinais e achados anormais de exames clínicos e de & 10 & 5,26 \\
laboratório, não classificados em outra parte (R00-R99) & $\mathbf{9}$ & $\mathbf{4 , 7 4}$ \\
R10 Dor abdominal e pélvica & 9 & 4,74 \\
\hline Capitulo VI - Doenças do sistema nervoso & $\mathbf{8}$ & $\mathbf{4 , 2 1}$ \\
G91 Hidrocefalia & 6 & 3,15 \\
\hline Capitulo XXI - Fatores que influenciam o estado de saúde e o contato com os & $\mathbf{4 4}$ & $\mathbf{2 3 , 1 6}$ \\
\hline serviços de saúde (Z00-Z99) & & \\
Z38 Nascidos vivos segundo o local de nascimento & &
\end{tabular}

Fonte: dados do SIH (2017).

No Capítulo XVIII são registradas as causas mal definidas, que totalizaram menos do que $6 \%$ dos internamentos. As hidrocefalias foram inclusas como doenças do sistema nervoso central em apenas $9(4,74 \%)$ internamentos. Interessante notar que o local de nascimento foi apontado no Capítulo XXI, como impactante na condução do nascimento e internação de 6 neonatos $(3,15 \%)$. 


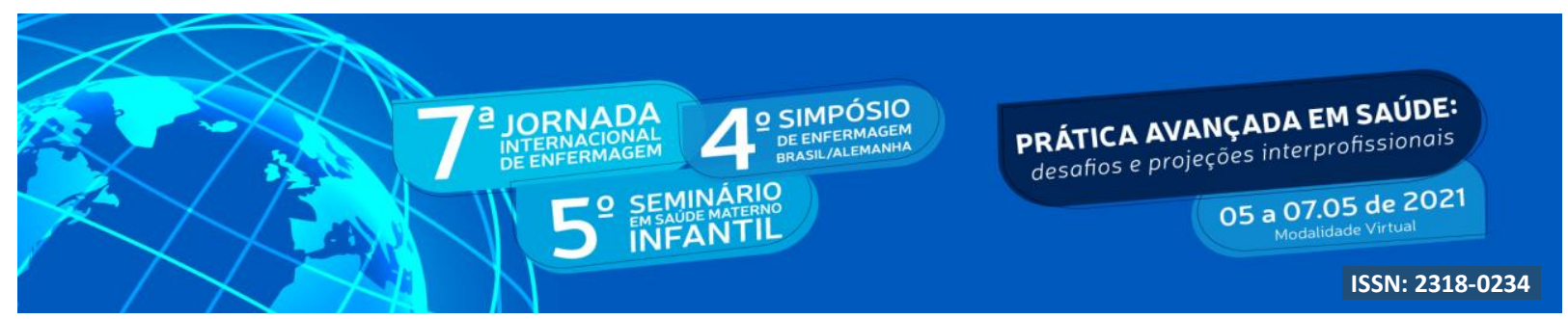

Evidenciou-se a prevalência da prematuridade e do baixo peso ao nascer, nas internações ocorridas na UCINCa: 10,7\% foram prematuros extremos e 13\% de extremo baixo peso. Este resultado é corroborado com o estudo descritivo, realizado no Rio Grande do Norte, que analisou 70 prontuários de recém-nascidos internados em UTIN e identificou incidência de $21 \%$ de prematuros com extremo baixo peso $(<1.000 \mathrm{~g})$ (OLIVEIRA; et al., 2013). Segundo a OMS, ocorrem 15 milhões de nascimentos prematuros por ano, com idade gestacional entre 32 a 37 semanas (80,0\%) (WHO, 2012). Observa-se concordância com o cenário internacional, devido aumento da prematuridade e baixo peso, os quais necessitam de tecnologia e de cuidados intensivos especializados, para a manutenção da vida.

Identificam-se múltiplas causas de internações em UTIN para a sobrevivência de recém-nascidos, permanecendo longos períodos internados. Estudo documental, realizado em UTIN, no Sul do Brasil, relacionou as infecções, distúrbios respiratórios e insuficiência renal como desfechos relacionado há um longo período de internação (ARRUÉ; et al., 2013). Na tentativa de redução do impacto da internação de prematuros, foi implantado o Método Canguru, que proporcionou maior estabilidade clínica e menor tempo de internação, fatos consequentes ao vínculo entre a mãe e recém-nascido mediante o contato pele a pele, que favorece expressivamente a promoção do aleitamento materno. E ainda, impacta na minimização da dor, do stresse e do risco de infecção neonatal (GESTEIRA; et al., 2013).

A redução do tempo de internação pode estar relacionada à alta precoce, recomendada ao atingirem 1.600g (BRASIL, 2011). Na presente pesquisa, 84,8\% dos recém-nascidos receberam alta hospitalar, destes $49,1 \%$ tinham peso menor que $2.000 \mathrm{~g}$, coerente à recomendação do Ministério da Saúde. Com destaque para o período de internação com média de 6 dias, entretanto este período é particular à cada neonato, e está relacionado à idade gestacional e o peso do nascimento. Por isso, na continuidade do cuidado e apoio às famílias, é necessário que estejam vinculados à terceira etapa do Método Canguru. O seguimento permitirá a observação do ganho de peso e está relacionado às orientações para que a mãe realize os cuidados de forma segura em domicílio. O cuidado deverá estar apoiado no contato pele a pele, na promoção do aleitamento materno e, associado ao acompanhamento ambulatorial, durante as primeiras 48h após a alta, para monitoramento (BRASIL, 2011). 


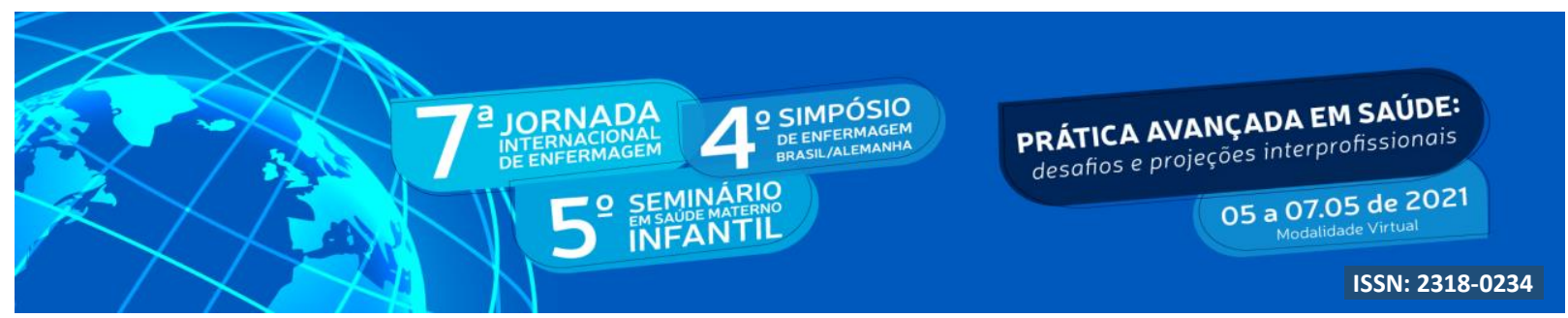

Estudo descritivo realizado nos Estados Unidos, em 2015, apontou redução de $8 \%$ de gestações na adolescência (15 a 19 anos), em relação a 2014. Entretanto, para mulheres acima de 30 anos, houve aumento de $1 \%$, e acima 40 anos, o aumento foi para $4 \%$. Contexto que está relacionado a maior incidência de nascimentos entre mulheres de 20 a 30 anos (MARTIN; et al., 2017), corroborando os dados do presente estudo. O artigo apontou que 9,6\% dos nascidos vivos foram prematuros, com idade gestacional entre 32 a 37 semanas; que houve aumento de recém-nascidos com baixo peso ao nascer, mantendo taxas estáveis para o extremo baixo peso $(<1.500 \mathrm{~g})$ e, elevação entre 1.500 a $2.499 \mathrm{~g}$, estando o peso relacionado intimamente com a idade gestacional (MARTIN; et al., 2017), resultados semelhantes ao identificado nesta pesquisa.

Os índices de cesarianas nos EUA decresce, o nascimento cirúrgico é escolhido por mulheres com 40 anos ou mais (MARTIN; et al., 2017). Diferindo-se da presente pesquisa, que identificou a cesariana em 61,3\% dos nascimentos, e parto normal em 34,5\%. No Brasil, as taxas de cesarianas, estão acima (RISCADO; et al., 2016) do que é preconizada pela OMS, entre 25 a $30 \%$. A escolha por esse tipo de parto pode estar relacionada ao critério médico, principalmente, pela falta de profissionais qualificados para a assistência ao parto vaginal (BRASIL, 2015). Outros motivos que levam as próprias mulheres a escolherem esta via, são situações de ausência de acompanhante, demora no tempo de admissão, experiências ruins na família ou da própria parturiente, medo, falta de técnicas para alivio da dor e ideias de que o parto vaginal é arriscado para a mãe e para o bebê, além da cultura do planejamento do nascimento ao possibilitar a escolha da data (RISCADO; et al., 2016).

Os dados do estudo em relato, aponta para elevada incidência de gestação única (87\%) em comparação a gemelaridade (13\%). Estudo nacional, explica que no Sistema de Informação sobre Nascidos Vivos, em 2013, nasceram 2.904.027, destes 97,9\% foram gestação única e apenas 2,1\% gemelar. A gestação de gemelares pode desencadear alguns fatores de risco para a mãe e para o feto, complicações durante a gestação como a préeclâmpsia com maiores índices de mortalidade perinatal, prematuridade, malformações fetais e restrição de crescimento intra-uterino (ANJOS; BOING, et al., 2013). A maior parte das gestantes eram casadas ou possuíam união estável (47,0\%), este fato mostra-se relevante, visto que a presença do companheiro no momento da gestação favorece a estabilidade 


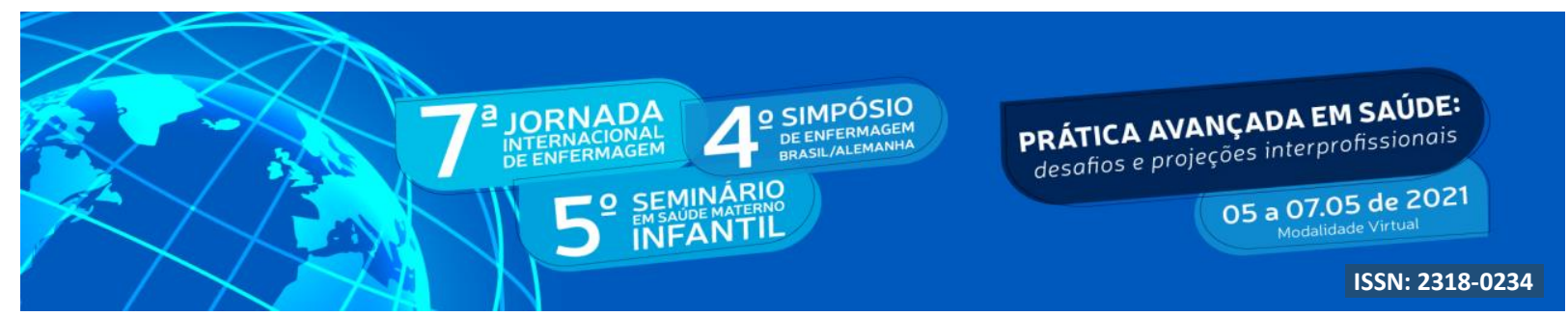

emocional da mulher, por ofertar apoio e segurança, favorecendo a adesão ao acompanhamento pré-natal (RENNER; et al., 2015).

Como principal limitação deste estudo observou-se a grande proporção de perda de dados devido à ausência dos registros maternos e dos neonatos. Destacam-se as perdas referentes as variáveis maternas escolaridade, estado civil e número de consultas de pré-natal são significativas, pois se enquadram na faixa muito ruim (50\% ou mais), ruim (20 a 50\%) respectivamente, conforme a avaliação de Romero e Cunha (2007). Outro estudo realizado em Pelotas, avaliou os registros do Sistemas de Informação Hospitalar e também identificou falta de informações registradas no sistema. Este fato ocasiona inconsistência nos indicadores de qualidade e, divergências pela falta de padronização e/ou erros na coleta destas informações. Para tanto, a capacitação profissional para o correto registro de dados se faz necessária para a garantia da qualidade dos dados informados (RASIA; et al., 2012).

\section{CONCLUSÃO}

A UCINCa assistiu recém-nascidos prematuros, de baixo peso ao nascer, adequados para a idade gestacional. Atenta-se para a alta prevalência de encaminhamentos, por se tratar de um hospital de referência estadual de alto risco. Sugere-se que outros estudos possam analisar as habilidades e a segurança adquirida pelas mães para a realização do cuidado a seus filhos prematuros e de baixo peso em seu domicílio, após a alta hospitalar. A principal limitação deste estudo é intrínseca ao trabalho com dados secundários, sobretudo devido a proporção de perda dos dados. Há fragilidade no preenchimento das variáveis socioeconômicas e número de consultas de pré-natal, realizado pela equipe médica do hospital, reitera-se a importância da capacitação destes profissionais para o correto registro dos dados e conquista de maior segurança para o paciente/binômio, profissional de saúde e instituição.

\section{REFERÊNCIAS}

WHO. World Health Organization. Born too soon: the global action report on preterm birth. Geneva; 2012.

STELMAK, A. P; FREIRE, M. H. S. Aplicabilidade das ações preconizadas pelo método canguru. Rev Fund Care Online. n.9, v.3, p. 795-802, 2017. 


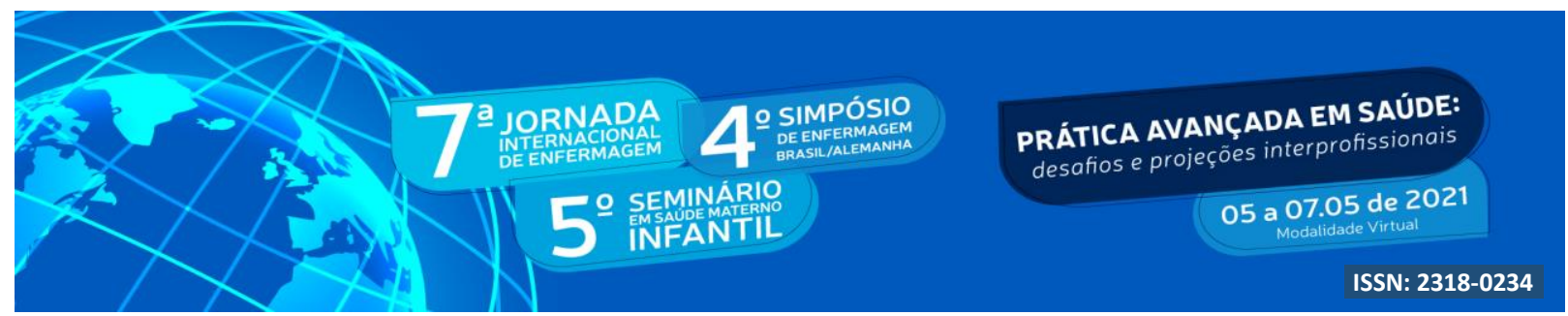

BRASIL. Ministério da Saúde. Atenção à saúde do recém-nascido: guia para os profissionais de saúde. Brasília-DF, 2011.

SOUSA, D. S. ET. AL. Morbidade em recém-nascidos prematuros de extremo baixo peso em unidade de terapia intensiva neonatal. Rev. Bras. Saude Matern. Infant. v. 17, n. 1, 2017.

ARAGÃO, J. Introdução aos estudos quantitativos utilizados em pesquisas cientificas. Revista Práxis. v. 3, n. 6, 2011.

MALTA M. ET AL. Iniciativa STROBE: subsídios para a comunicação de estudos observacionais. Rev Saúde pública. n. 44, v. 3, p.559-65, 2010.

OLIVEIRA, C. O. P. ET AL. Caracterização das infecções relacionadas à assistência à saúde em uma unidade de terapia intensiva neonatal. Rev Enferm. n. 21, v. 1, p. 1577-85, 2013.

ARRUÉ, A. M. Caracterização da morbimortalidade de recém-nascidos internados em unidade de terapia intensiva neonatal. Rev Enferm. n. 3, v. 1, p. 86-92, 2013.

GESTEIRA, E. C. R. ET AL. Método canguru: benefícios e desafios experienciados por profissionais de saúde. Rev Enferm. n. 6, v. 4, p. 518-28, 2016.

MARTIN, J. A. ET AL. National Vital Statistics Reports. n. 66, v. 1, 2017.

RISCADO, L. C. ET AL. A decisão pela via de parto no Brasil: Temas e tendências na produção da saúde coletiva. Texto Contexto Enferm. n. 25, v. 1, p. e3570014, 2016.

BRASIL. Ministério da Saúde. Comissão Nacional de Incorporação de Tecnologias no SUS. Diretrizes de atenção à gestante: a operação cesariana. Protocolo. Brasília, 2015.

ANJOS, J. C; BOING, A. F. Diferenças regionais e fatores associados ao número de consultas de pré-natal no Brasil: análise do Sistema de Informações sobre Nascidos Vivos em 2013. Rev Bras Epidemiol. n. 19, v. 4, p. 835-50, 2016.

RENNER, F. W. ET AL. Perfil epidemiológico das puérperas e dos recém-nascidos atendidos na maternidade de um hospital de referência do interior do Rio Grande do Sul no primeiro semestre de 2014. Boletim Científico de Pediatria. n. 4, v. 2, p. 27-32, 2015.

RASIA, I. C. R. B. ET AL. A utilização dos sistemas de informação em uma instituição de saúde de Pelotas/RS. Rev. Saúde. n. 8, v. 2, p. 32-42, 2012.

ROMERO, D. E; CUNHA, C. B. Avaliação da qualidade das variáveis epidemiológicas e demográficas do Sistema de Informação sobre Nascidos Vivos, 2002. Cad. Saúde Pública. n. 23, v.3, p. 701-14, 2007. 